\title{
O LORI como método de avaliação de objetos de aprendizagem: estudo de revisão
}

\author{
Maicon Tavares de Souza ${ }^{1}$ \\ Rodrigo Marcelino ${ }^{2}$ \\ Ivan Fortunato ${ }^{3}$
}

\section{Resumo}

Este artigo tem como objetivo realizar uma pesquisa do estado da arte sobre a utilização do LORI como método de avaliação de Objetos de Aprendizagem, delimitando o período dos últimos cinco anos. Para isso foi utilizado o Google Acadêmico como local pesquisas para encontrar artigos científicos que sejam adequados à proposta de trabalho. Os dados encontrados demonstram que o uso desta ferramenta ainda é pouco expressivo, tendo em vista a pequena quantidade de trabalhos publicados, apesar da grande importância que diversos autores atribuem à qualidade dos Objetos de Aprendizagem. Ao final, espera-se que este artigo possa, de alguma maneira, auxiliar educadores, profissionais da informática e demais pessoas no estudo e reflexão sobre o tema.

Palavras-chave: LORI. Informática aplicada à educação. Avaliação. Objetos de Aprendizagem.

\begin{abstract}
This paper aims to carry out a state-of-the-art research on the use of LORI as a method of evaluating Learning Objects, delimiting the period of search back five years. For this we used the Google Scholar as a local for search in order to find scientific papers that are appropriate to the proposal. The data show that the use of the LORI as an evaluating tool is still not very expressive, considering the small amount of published papers, despite the great importance that several authors attribute to the quality of Learning Objects. At the end, it is hoped that this paper can, in some way, help educators, computer professionals and other people in the study and reflection on the topic.
\end{abstract}

Kenwords: LORI. Informatics applied to education. Evaluation. Learning objects.

\footnotetext{
${ }^{1}$ Especialista em Informática aplicada à Educação do IFSP Itapetininga. Graduado em análise e desenvolvimentos de sistemas pela FATEC Itapetininga. Contato: maikonitap@gmail.com.

${ }^{2}$ Especialista em Informática aplicada à Educação do IFSP Itapetininga. Graduado em análise e desenvolvimentos de sistemas pela FATEC Itapetininga. Contato: rodrigo.marcelino@fatec.sp.gov.br.

${ }^{3}$ Doutor em Desenvolvimento Humano e Tecnologias e Doutor em Geografia, ambos pela Universidade Estadual Paulista Júlio de Mesquita Filho, Rio Claro. Pós-doutorado em Ciências Humanas e Sociais pela Universidade Federal do ABC. Professor do curso de especializaçáo em Informática aplicada à Educação do IFSP Itapetininga. Docente permanente do Programa de Pós-Graduação em Educação da UFSCar, campus Sorocaba. Contato: ivanfrt@ yahoo.com.br.
} 
Este artigo foi produzido a partir de um trabalho de conclusão de curso de pós-graduação lato senso em Informática aplicada à Educação, que trata de um levantamento do tipo estado da arte de artigos nacionais publicados nos últimos cinco anos que discutem a avaliação de objetos de aprendizagem, com foco específico em um dos métodos mais indicado e utilizado para esse fim, o LORI (Learning Object Review Instrument).

Nos últimos anos, temos visto grande crescimento do uso das tecnologias digitais de comunicação e informação nas mais diversas áreas da sociedade, inclusive na educação. Mas, na escola, sua presença tem se desenvolvido de maneira tímida, encontrando resistências de vários tipos (recursos, gestão, leis, docentes, discentes etc.). No caso específico dos docentes, um dos motivos de resistência, explicam Braga et al. (2012), é a falta de preparo, sendo a maneira "tradicional" de ensino a forma mais factível de ação (falta de tempo, de recursos na escola, salas lotadas etc.).

Nesse cenário, as ferramentas mais acessíveis, capazes de minimizar resistências, são os Objetos de Aprendizagem, ora referidos apenas como OA. Estes são desenvolvidos e estáo disponíveis para todos os meios tecnológicos digitais utilizados no ensino, em todas as áreas do conhecimento. Segundo Gama (2007), o desenvolvimento e a avaliação dos OA tem sido um grande desafio, pois é difícil saber se um objeto de aprendizagem é eficaz e atende as necessidades educacionais para qual foi projetado. Apesar das facilidades de uso e acesso, Cechinel et al. (2012) afirmam que ainda não há um consenso para definir qual a melhor abordagem de avaliação de um OA, pois existem diversas abordagens. Uma das dificuldades desse processo é a definição da qualidade para um OA.

Segundo Chacon et al. (2012 apud SILVA et al., 2016), as principais abordagens para avaliar um objeto de aprendizagem são: Método Reeves, Técnica de Mucchielli, Técnica de Inspeção de Conformidade Ergonômica de Software Educacional (TICESE) e o LORI. Este método foi desenvolvido, no ano de 2002, pela e-Learning Research and Assessment Network (eLera) com o propósito de se tornar o instrumental mais adequado para avaliação de um OA pelos próprios professores. Esse instrumento consiste na avaliaçáo de nove itens, utilizando uma escala de cinco pontos. Esses itens são descritos como: qualidade de conteúdo; alinhamento do objetivo da aprendizagem; feedback e adaptação; motivação; design da apresentação; usabilidade; acessibilidade; reusabilidade; e aderência e padróes. $\mathrm{Na}$ avaliação dos $\mathrm{OA}$, esses itens recebem uma nota dentro de uma escala que vai de uma a cinco estrelas, quanto maior a pontuação mais eficiente ele é. Importante destacar que, se este item não se aplicar ao objeto em questão então ele recebe uma nota N/A (não se aplica).

Dessa forma, como o propósito desta pesquisa é apresentar um estado de arte da produção nacional, abordando o assunto de avaliação de Objetos de Aprendizagem com utilização do método LORI, analisando como este vem sendo utilizado nos últimos cinco anos. O local de pesquisa foi delimitado ao repositório do Google Acadêmico, local de buscas mais práticos que outros repositórios, sendo utilizado, portanto, não apenas na pesquisa acadêmica, mas na busca cotidiana por soluçóes mais consubstanciadas. Para alcançar esse objetivo, o artigo foi desenvolvida em três partes, sendo que, na primeira apresentamos uma discussão sobre Objetos de Aprendizagem, como eles são definidos por alguns autores, suas principais características e quais são os elementos fundamentais para garantir um bom aproveitamento em sua utilização no ensino. Apresentada as 
definiçóes e os elementos essenciais, são apresentadas, por meio de repositórios e sites, maneiras de obter acesso a esses OA.

$\mathrm{Na}$ segunda parte, foi realizada uma pesquisa de como a avaliação de OA é definida, quais as maneiras que pode ser feita e como os pesquisadores definem os aspectos mais importantes a serem levados em consideração. Ainda nesta parte, estudamos mais detalhadamente o funcionamento do método LORI juntamente com um exemplo de como uma avaliação pode ser realizada por meio deste instrumento. $\mathrm{Na}$ terceira e última parte descrevemos quais os métodos utilizados para a realização deste trabalho, bem como os dados que foram coletados sobre a produção acadêmica referente ao tema. Por fim apresentamos a análise destes dados e as consideraçóes finais da pesquisa.

Ao final, espera-se que este trabalho de sistematização parcial da literatura colabore com o estado da arte a respeito de avaliaçóes de OA, e que novas buscas sejam realizadas, seja por educadores, programadores e/ou usuários desses objetos.

\section{Parte 1. Sobre Objetos de Aprendizagem}

De acordo com Braga et al. (2012), a maioria dos recursos digitais utilizados no aprendizado são chamados de OA. Guillermo, Tarouco e Endres (2005) consideram Objetos de Aprendizagem como elementos de uma nova metodologia de ensino e aprendizagem, baseada no uso do computador e da Internet. Para Galafassi et al. (2014), os OA oferecem a possibilidade de auxiliar na aprendizagem mediada por computador, organizando e, ao mesmo tempo, trazendo mais riqueza semântica aos conteúdos educacionais digitais. Wiley (2000 apud BARBOSA, 2014, p. 34), por sua vez, considera que um "Objeto de Aprendizagem é qualquer entidade digital ou não digital, que possa ser usada, reusada ou referenciada durante o ensino com suporte tecnológico".

Segundo Braga et al. (2012, p. 3), os Objetos de Aprendizagem devem possuir as seguintes características de uso e aprendizagem: (a.) Habilidades didático pedagógicas: o aprendizado proposto deve estar claro ao usuário, bem como o OA deve oferecer feedback suficiente sobre o desenvolvimento da aprendizagem; (b.) Acessibilidade: esta é uma característica importante dos OA, pois eles devem possuir uma versão que atenda a diversos tipos de usuários, como jovens, adultos, crianças, idosos, assim como portadores de necessidades específicas ou com algum tipo de deficiência. É importante também ser acessado por diferentes dispositivos, não importando o tipo e velocidade de conexão utilizada; (c.) Precisáo: o OA precisa sempre atingir seus objetivos conforme o planejamento; (d.) Disponibilidade: o OA precisa ser encontrado facilmente e, para isso, dever ser ordenado como um índice e armazenado corretamente; (e.) Confiabilidade: falhas técnicas ou erros em seu conteúdo pedagógicos não devem ser tolerados por um $\mathrm{OA}$; (f.) Usabilidade: o OA não deve apresentar dificuldade em sua utilizaçáo e precisa seguir os padróes de usabilidade que estejam consagrados na atualidade; (g.) Portabilidade: para atender a esta característica, o OA não dever falhar em diferentes contextos como: tipos de hardware (tablets, celulares, computadores etc.) e em diferentes sistemas operacionais; (h.) Facilidade de instalaçáo: o OA dever ser instalado facilmente; (i.) Interoperabilidade: se houver necessidade, o OA dever ser capaz de exportar seus dados para outro tipos de sistema. 


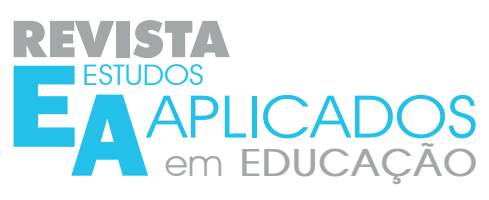

Aguiar e Flôres (2014) afirmam que a utilização de um OA como ferramenta de ensino apresenta muitas vantagens, podendo ser aplicada em diversas áreas da educação, tornando-se um ótimo auxilio para o docente em sala de aula. Sua apresentação pode ser em vários formatos, como apresentação de vídeos, slides, animação entre outros. Para os autores, "devido ao seu potencial de reusabilidade, durabilidade e adaptabilidade, os OAs são materiais educacionais com os quais o aluno pode interagir, sendo coautor de sua aprendizagem" (AGUIAR; FLÔRES, 2014, p. 25).

É muito importante salientar, conforme Costa (2014), que os objetivos pedagógicos devem ser levados em consideração na escolha do objeto de aprendizagem e também aspectos como: a faixa etária, interatividade, motivação dos alunos para o conhecimento e um fator muito importante, se o OA atende o objetivo de contribuir para o ensino.

Existem diversos "locais" na internet onde um professor pode ter acesso a uma variedade enorme de OA, sendo os mais populares:

(a.) Phet (<https://phet.colorado.edu/pt_BR/>): plataforma Web, disponível em português, desenvolvida pela universidade do Colorado nos EUA que disponibiliza diversas simulações de ciências e matemática auxiliando professores e alunos de todas as idades;

(b.) KIDUCA (<http://www.kiduca.com.br>): plataforma educacional brasileira que, através de games, ajudam no aprendizado de diversas disciplinas do currículo escolar;

(c.) Khan Academy (<https://pt.khanacademy.org/>): site que oferece diversos exercícios e vídeo aulas em diversas disciplinas, como: matemática, ciências, programação de comutadores, história etc.;

(d.) Scratch (<https://scratch.mit.edu>): projeto desenvolvido pelo MIT (Massachusetts Institute of Technology), que se trata de uma plataforma web e também um software que pode ser instalado, que trabalha o ensino da programação de jogos, animaçôes e também raciocínio lógico.

Nos diferentes repositórios de OA, não é difícil encontrar o tema educacional que se procura. Podemos citar alguns exemplos, como o RIVED, ou Rede Interativa Virtual de Educaçáo (rived. mec.gov.br). Trata-se de um programa da Secretaria de Educação a Distância (SEED), que disponibiliza diversos OA, especialmente voltados ao ensino de Ciências. Temos, também, o Portal do Professor (portaldoprofessor.mec.gov.br) que, além de disponibilizar diversos OA, também oferece um excelente suporte educacional aos professores, bem como proporciona uma troca de experiências e compartilhamento de conhecimentos. Outro exemplo é o Banco Internacional de Objetos Educacionais ${ }^{4}$, sendo que este é mais um projeto da SEED que apresenta centenas de conteúdos com diversos formatos como áudio, vídeo, animaçóes, mapas, softwares educacionais entre outros. Esse repositório apresenta recursos de diversos países e línguas, assim pessoas de qualquer parte do mundo poderão acessar.

Contudo, é necessário ter cuidado ao selecionar um OA, pois nem todos seguem os conceitos educacionais necessários para uma boa aprendizagem e nem todos são desenvolvidos por profissionais da área da computação, conforme explicam Braga et al. (2014, p. 2):

${ }^{4}$ Site: <http://objetoseducacionais.mec.gov.br>. 
No âmbito da educaçáo, o uso de OA que náo cumprem os objetivos pode ser algo catastrófico, pois podem ensinar erroneamente um determinado conteúdo. Já no âmbito computacional, um OA que possui problemas técnicos pode contribuir para a desmotivação do aluno. Um OA ideal seria aquele que tivesse um equilíbrio técnico e pedagógico e que pudesse ser frequentemente reutilizado e contribuir de maneira efetiva para o aprendizado.

Por isso, é fundamental que os professores saibam não apenas como acessar uma base com inúmeros $\mathrm{OA}$, mas, que tenham consciência de que não é apenas possível, como é necessário avaliar um OA antes de recomendar seu uso aos seus estudantes. Assim, dedicamos a seção seguinte para discutir com mais propriedade a avaliação de Objetos.

\section{Parte 2. Sobre Avaliação de AO}

Segundo Santos e Amaral (2012), através dos OA podemos intensificar a aprendizagem. Assim, como os OA apresentam grande potencial para serem utilizados como ferramenta de apoio ao ensino, com isso, é fundamental realizar uma avaliação criteriosa e entender em qual teoria de aprendizagem ele está inserido, pois, conforme afirmam Bulegon e Mussoi (2014), em muitos casos nem mesmo seu desenvolvedor. Para Almeida et al. (2012) é necessária uma maior discussão acerca da qualidade de OA e, para que haja um melhor aproveitamento na utilização, os professores precisam realizar uma avaliação profunda destes objetos para obter um ótimo aproveitamento em suas práticas pedagógicas. Encontramos, na literatura, diversas metodologias de avaliação de $\mathrm{OA}$, buscando aquela que seja mais eficaz na área educacional. Dessa forma, o objetivo é permitir que os docentes possam escolher os melhores recursos para auxiliar no ensino.

Cechinel (2014) afirma que não há um consenso para uma definição sobre a qualidade de um Objeto de Aprendizagem, tornando a avaliação uma tarefa árdua, que geralmente envolve diferentes aspectos, tais como interpretação do avaliador sobre os critérios e valores (por exemplo, usabilidade, qualidade de conteúdo etc.) e o público alvo para o qual o objeto foi projetado. Entretanto, Bethard et al. (2009 apud CECHINEL, 2014) declaram que a qualidade de um OA precisa levar em consideração o contexto da situação e depende do "alinhamento entre o grupo de usuários sendo servidos pelo objeto, a ambientação em que o objeto foi implementado, e o propósito designado para o objeto" (p. 73).

Para Reategui e Finco (2010), a avaliação de OA é dividida em duas partes, sendo uma sobre seus aspectos pedagógicos e a outra sobre aspectos técnicos. $\mathrm{O}$ primeiro trata-se da preocupação em saber se o OA foi desenvolvido de acordo com a proposta pedagógica escolhida pelo desenvolvedor. O segundo diz respeito às características técnicas necessárias, que fazem com que o OA tenha qualidade de acordo com as diretrizes do método de avaliação, neste caso utilizando o LORI. 
De acordo com Williams (2000 apud CECHINEL, 2014), "o que um objeto de aprendizagem deve ser, está relacionado com as perspectivas das diferentes opiniôes daqueles são os verdadeiros usuários do recurso" (p. 74). Logo, o autor propóe que sua avaliação deva abranger todo processo de criação do objeto. Assim, a avaliaçáo pode ser realizada por vários tipos de pessoas, diretamente interessadas no recurso, como professores, alunos, desenvolvedores, intituiçôes etc. O autor também afirma que, para a avaliação de um OA que esteja pronto para ser utilizado pelo seu usuário final, que vai aprender com o objeto, o método LORI (Learning Object Review Instrument) é o mais reconhecido.

Sobre o LORI, Medeiros e Schimiguel (2012) afirmam que este instrumento possui os seguintes critérios para avaliação:

(a.) Qualidade de conteúdo: aqui, um dos aspectos mais importantes, pois diz respeito à veracidade e precisão das informações, abordando se há erros e equilíbrio nas ideias;

(b.) Alinhamento do bjetivo da aprendizagem: está ligado com o nivelamento entre as atividades e o objetivo proposto pelo OA, observando se os usuários conseguem adquirir o conhecimento necessário para responder positivamente à avaliação;

(c.) Feedback e adaptaçáo: este item mede a capacidade do OA de se adaptar às necessidades do usuário e oferecer um feedback daquilo que foi proposto como objetivo;

(d.) Motivaçáo: trata-se da capacidade de motivar o usuário no desenvolvimento das atividades e também conseguir reter sua atenção.;

(e.) Design da apresentaçáo: temos, neste item, uma avaliação da qualidade da exposição dos elementos visuais do OA, como, por exemplo, os elementos de vídeos, gráficos, textos etc.;

(f.) Usabilidade: temos aqui uma forma de avaliar a facilidade de usuário em navegar e utilizar os recursos do OA;

(g.) Acessibilidade: é a forma de avaliar se o Objeto de Aprendizagem se adapta a diferentes necessidades de pessoas com algum tipo de deficiência, e também de verificar a facilidade de utilizaçáo, independentemente do tipo de plataforma (sistema operacional), e dispositivo (tablet, smartphone ou computador);

(h.) Reusabilidade: capacidade de utilização do $\mathrm{OA}$ em diferentes contextos, por exemplo, em cursos e disciplinas distintas;

(i) Aderência e padróes: verificação do atendimento de normas e padrões internacionais de desenvolvimento.

Para a avaliação de um OA pelo LORI, cada um dos itens é verificado por meio de uma escala que vai de uma à cinco estrelas, sendo anotada a sigla "N/A" (não se aplica), para o item que não for relevante para o objeto (figura 01). Subentende-se que, nesta classificação, cinco estrelas significa que o critério foi plenamente atendido e que uma estrela significa que o critério não foi atendido, em absoluto. 


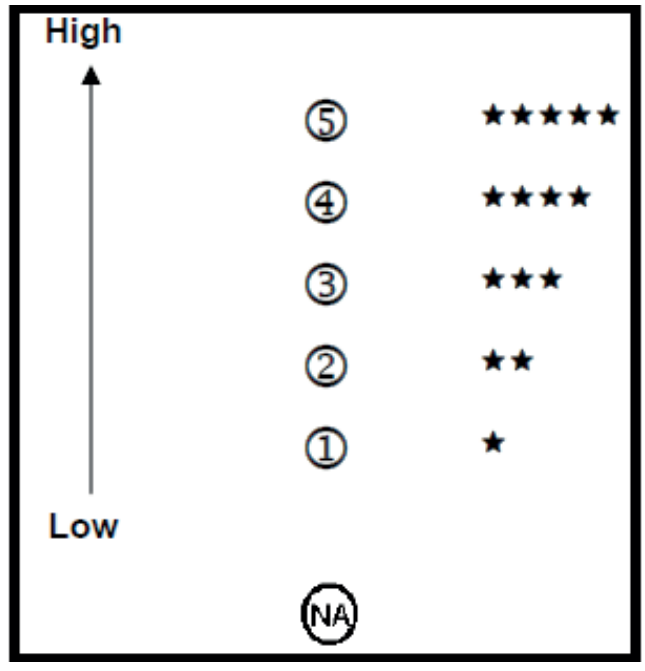

Figura 1 - Escala de Valores

Fonte: LORI User Manual 1.5

Vejamos, na sequência, um exemplo de utilização do método LORI, para avaliar o reconhecido OA chamado "Duolingo". Trata-se de um aplicativo para o ensino de idiomas disponível para diversas plataformas, tais como ANDROID, IOS, WINDOWS PHONE e WEB. Para construção do exemplo, escolhemos o ensino do Inglês utilizando o aplicativo na versão Android 7.0. Para facilitar a visualização do uso do LORI como ferramenta de avaliação de um OA, elaboramos o quadro 01, apresentando cada critério, descrevendo sua avaliação para o caso particular do "Duolingo", incluindo a quantidade de estrelas atribuídas para cada item.

Quadro 1 - Exemplo de avaliação de um OA pelo método LORI

\begin{tabular}{|c|c|c|}
\hline Critério & Avaliação & Qualificaçáo \\
\hline $\begin{array}{l}\text { Qualidade de } \\
\text { conteúdo }\end{array}$ & $\begin{array}{l}\text { A apresentação do conteúdo não demonstra nenhum } \\
\text { erro formal, as informações são claras e as instruções são } \\
\text { precisas aos usuários. }\end{array}$ & $\Delta \star \Delta t \Delta t$ \\
\hline $\begin{array}{l}\text { Alinhamento } \\
\text { do objetivo da } \\
\text { aprendizagem: }\end{array}$ & $\begin{array}{l}\text { As atividades propostas, conteúdo e avaliação são } \\
\text { coerentes com o objetivo proposto pelo OA, que é } \\
\text { ensinar um idioma. O objeto, por si só, é suficiente } \\
\text { para auxiliar o usuário a alcançar seu objetivo de } \\
\text { aprendizagem. }\end{array}$ & 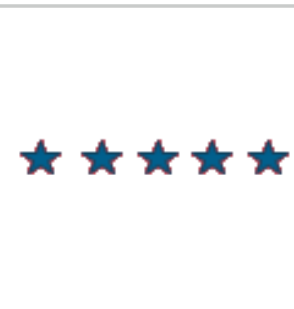 \\
\hline $\begin{array}{l}\text { Feedback e } \\
\text { adaptação }\end{array}$ & $\begin{array}{l}\text { O OA inicialmente propóe um teste de nivelamento para } \\
\text { averiguar os conhecimentos e, assim, adaptar o conteúdo } \\
\text { de ensino às necessidades do aluno usuário. Também } \\
\text { é possível acompanhar o progresso de aprendizagem } \\
\text { através do feedback do aplicativo. }\end{array}$ & 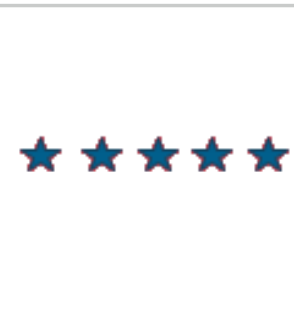 \\
\hline Motivação & $\begin{array}{l}\text { Para cada atividade concluída, o aluno aumenta sua } \\
\text { pontuação e o nível de aprendizado; além disso, são } \\
\text { propostos alguns exercícios em forma de desafio. }\end{array}$ & 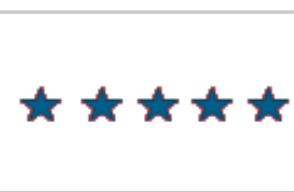 \\
\hline
\end{tabular}




\begin{tabular}{|c|c|c|}
\hline $\begin{array}{c}\text { Design da } \\
\text { apresentação }\end{array}$ & $\begin{array}{l}\text { Os elementos gráficos estão bem dispostos dentro da } \\
\text { plataforma e bem rotulados, a apresentação do texto } \\
\text { bem legível facilitando a aprendizagem. O ambiente } \\
\text { também é agradável com a apresentação das cores. O que } \\
\text { incomoda são os anúncios mostrados na versão gratuita. }\end{array}$ & tht \\
\hline Usabilidade & $\begin{array}{c}\text { A utilização e navegação do OA são bem simples e } \\
\text { intuitivas, com instruçóes bem claras. }\end{array}$ & $x \times x \times x$ \\
\hline Acessibilidade & $\begin{array}{l}\text { Como estamos utilizando a versão aplicativo, o acesso } \\
\text { as funções são bem atendidas através do toque na tela. } \\
\text { No entanto, sentimos a falta de um menu de ajuda ao } \\
\text { usuário. A versão Web, por sua vez, possui outra forma } \\
\text { de funcionamento, por meio de mouse e teclado, o que } \\
\text { pode limitar usuários com alguma deficiência. }\end{array}$ & 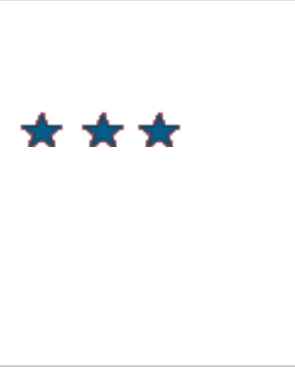 \\
\hline Reusabilidade & $\begin{array}{l}\text { Nesta avaliaçáo, identificou-se que o OA somente pode } \\
\text { ser usado no ensino de idiomas, não foi encontrado } \\
\text { outro contexto ou situaçáo para reaproveitamento. }\end{array}$ & $x t$ \\
\hline $\begin{array}{l}\text { Aderência e } \\
\text { padróes }\end{array}$ & $\begin{array}{l}\text { Não foi identificada a necessidade do OA se } \\
\text { adequar a alguma norma técnica ou padrão, como } \\
\text { por exemplo do IEEE (Institute of Eletrical and } \\
\text { Electronics Engineers). }\end{array}$ & N/A \\
\hline
\end{tabular}

\section{Parte 3. Revisão sistemática no Google Acadêmico}

Nesta seção, buscou-se levantar a produção acadêmica a respeito do LORI como método de avaliação de OA. Para tanto, selecionamos o repositório do Google Acadêmico como local de pesquisa, e utilizamos os seguintes descritores para busca: “LORI” AND “Objetos de Aprendizagem”. Inicialmente, tivemos como resultado 121 artigos publicados.

Porém, com a necessidade de atestar a atualidade sobre o objeto pesquisado, determinou-se que fosse aplicado um filtro de busca delimitando o período de 2012 a 2017, retornando um total de 87 artigos. Ainda assim, foi especificado que seriam somente artigos publicados em "Português", resultando em 77 artigos encontrados. Para se analisar apenas artigos pertinentes ao tema, foram selecionados deste total sete artigos, para isso, foram lidos os resumos destes trabalhos para observar quais abordavam diretamente a "Avaliação de Objetos de Aprendizagem" em seu escopo. Destaca-se, nesta análise, a ausência de artigos pertinentes nas publicaçóes em 2017, porém, não é de se alarmar, tendo em vista que este inventário foi feito durante o mesmo ano corrente. Todas essas filtragens estão contempladas no quadro 02 , a seguir. 
Quadro 2 - Total de artigos pertinentes por ano de publicação.

\begin{tabular}{|c|c|c|c|}
\hline Ano & Artigos Publicados & Artigos Pertinentes & Porcentagem (\%) \\
\hline 2012 & 11 & 2 & $18,2 \%$ \\
\hline 2013 & 9 & 1 & $11,1 \%$ \\
\hline 2014 & 17 & 1 & $5,9 \%$ \\
\hline 2015 & 20 & 2 & $10,0 \%$ \\
\hline 2016 & 17 & 1 & $5,9 \%$ \\
\hline 2017 & 3 & 0 & $0,0 \%$ \\
\hline Total & 77 & 7 & $\mathbf{9 , 1} \%$ \\
\hline
\end{tabular}

$\mathrm{Na}$ sequência, a fim de conhecer melhor os autores responsáveis pelas produções acadêmicas selecionadas, uma segunda etapa um levantamento de dados foi elaborada. Nesta, foram inventariadas informaçóes importantes sobre os autores, como suas universidades ou instituiçóes de ensino de origem, as quais foram discriminadas no quadro 03 a seguir.

Quadro 3. Disposição dos autores por Universidades a que pertencem.

\begin{tabular}{|c|c|c|c|c|c|c|}
\hline Instituiçáo & 2012 & 2013 & 2014 & 2015 & 2016 & 2017 \\
\hline Universidade Federal de Mato Grosso & & 3 & & & & \\
\hline Universidade Federal de São Carlos & & & 5 & & & \\
\hline Universidade Federal da Paraíba & & & & & 5 & \\
\hline Universidade de Sáo Paulo & & & & & 1 & \\
\hline Universidade de Pernambuco & & & & 3 & & \\
\hline Universidade Federal de Alagoas & & & & 3 & & \\
\hline Universidade Cruzeiro do Sul & 4 & & & 2 & & \\
\hline Universidade Pontifícia de Minas Gerais & & & & 1 & & \\
\hline Total & $\mathbf{4}$ & $\mathbf{3}$ & $\mathbf{5}$ & $\mathbf{9}$ & $\mathbf{6}$ & $\mathbf{0}$ \\
\hline
\end{tabular}

Ao analisar o Quadro 03, podemos observar uma distribuição interessante quanto ao tipo de instituição a qual pertencem os autores, divididas em: 04 (quatro) federais, 02 (duas) estaduais, uma (01) confessional e uma (01) particular. Também é possível ver as regiôes do Brasil onde estáo divididos os trabalhos: Sudeste, Centro-Oeste e Nordeste, sentimos falta das regióes Norte e Sul. Nenhuma instituição apresentou trabalhos dando continuidade no assunto, com exceção da Universidade Cruzeiro do Sul, que publicou em 2012 e 2015, porém com autores diferentes.

A formação acadêmica dos autores é também um item muito importante a ser considerado, isso para compreender quais setores estão estudando o método LORI nos últimos anos. O quadro 04 a seguir apresenta essa relação. 
Quadro 4 - Formação acadêmica dos autores e sua área de estudo.

\begin{tabular}{|c|c|c|c|}
\hline Autores & Nível & Área & Publicaçáo \\
\hline Márcio E. K. L. dos Santos & Doutorando & $\begin{array}{c}\text { Ensino de Ciências e } \\
\text { Matemática }\end{array}$ & 2012 \\
\hline Luiz Henrique Amaral & Especialista & Gestão Acadêmica & 2012 \\
\hline Maxwell de Oliveira Medeiros & Doutor & Ciência da Computação & 2012 \\
\hline Juliano Schimiguel & Doutor & Ciência da Computação & 2012 \\
\hline Laura A. F. Lima & Mestrando & Educação & 2013 \\
\hline Kátia M. Alonso & Doutor & Educação & 2013 \\
\hline Cristiano Maciel & Doutor & Computação & 2013 \\
\hline Marcos Tsuda & Bacharel & Ciência da Computação & 2014 \\
\hline Vinicius Moro Sanches & Bacharel & Ciência da Computação & 2014 \\
\hline Thalles Gonçalves Ferreira & Bacharel & Ciência da Computação & 2014 \\
\hline Joice Lee Otsuka & Doutora & Ciência da Computação & 2014 \\
\hline Delano Medeiros Beder & Doutor & Ciência da Computação & 2014 \\
\hline Wilk Oliveira dos Santos & Mestrando & Informática & 2015 \\
\hline Sebatião Rogério da Silva Neto & Mestrando & Informática & 2015 \\
\hline Clóvis Gomes da Silva Junior & Mestrando & Informática & 2015 \\
\hline Ig Ibert Bittencourt & Doutor & Ciência da Computação & 2015 \\
\hline Rosiney Rocha Almeida & Doutoranda & $\begin{array}{c}\text { Ensino de Ciências e } \\
\text { Matemática }\end{array}$ & 2015 \\
\hline Andréa Carla Leite Chaves & Doutora & $\begin{array}{c}\text { Ensino de Ciências e } \\
\text { Matemática }\end{array}$ & 2015 \\
\hline Carlos Fernando de Araújo Jr & Doutor & $\begin{array}{c}\text { Ensino de Ciências e } \\
\text { Matemática }\end{array}$ & 2015 \\
\hline Wendell S. Pereira & Licenciando & Ciência da Computação & 2016 \\
\hline Raimundo J. Cardoso Filho & Licenciando & Ciência da Computação & 2016 \\
\hline Williane Rodrigues de A. Silva & Mestrando & Sistemas de Informação & 2016 \\
\hline Raphael Salviano T. da Silva & Licenciando & Ciência da Computação & 2016 \\
\hline Vanessa F. Dantas & Mestre & Ciência da Computação & 2016 \\
\hline Yuska P. C. Aguiar & Doutora & Engenharia Elétrica & 2016 \\
\hline
\end{tabular}

Percebemos, pelo Quadro 04, que a área da Ciência da Computação predomina o tema de estudo desta pesquisa, com presença maior que a própria área da Educação, no qual aparece apenas três vezes na relação. Isso pode indicar que os educadores se atentam menos com avaliaçóes de OA do que profissionais de Informática, isto é, partindo do pressuposto de que o LORI é a principal ferramenta de avaliação de OA. Porém, para afirmar tal suposiçáo, pode ser necessário uma pesquisa posterior mais aprofundada sobre este assunto, o que fugiria do tema aqui proposto.

\section{Análise dos artigos}

Nesta parte da pesquisa, apresentamos uma síntese para cada, elaborada a partir da análise das questóes e hipóteses abordadas pelos respectivos autores. 
Artigo 01: Avaliação de objetos de aprendizagem: aspectos a serem considerados neste processo Ano de publicação: 2012

Autores: Rosiney R. Almeida; Andréa C. L. Chaves; Carlos Fernando de Araújo Jr.

Instituiçáa: Universidade Cruzeiro do Sul; Universidade Pontifícia de Minas Gerais

Síntese: Segundo os autores: "O presente trabalho tem como objetivo analisar e discutir alguns aspectos essenciais tratados por pesquisadores atuais na área do desenvolvimento e avaliação de objetos de aprendizagem" (ALMEIDA et al., 2012, p. 01). Neste artigo, os pesquisadores afirmam que há um grande crescimento na produçáo e divulgaçáo de OA com o propósito de auxiliar os docentes no processo de ensino. Contudo, os autores identificaram que há uma necessidade de verificar a qualidade pedagógica destes, e que muitas vezes eles são produzidos e divulgados sem passar por este processo. Os autores citam, ainda, que esses objetos deveriam passar por uma triagem avaliativa, tal qual a do método LORI, antes de serem disponibilizados em repositórios.

Artigo 02: Avaliação de objetos virtuais de aprendizagem no ensino de matemática Ano de publicação: 2012

Autores: Marcio Eugen Klingesnchmid Lopes dos Santos e Luiz Henrique Amaral Instituição: Universidade Cruzeiro do Sul

Síntese: Os autores trazem um artigo como parte de uma pesquisa de doutorado, com o seguinte objetivo: "analisar as características e contribuiçóes dos objetos virtuais de aprendizagem no ensino de matemática e sua importância como ferramenta pedagógica" (LOPES; AMARAL, 2012, p. 83). A pesquisa apresenta as principais definiçôes de OA e seus repositórios, mas também mostra como é importante utilizar métodos para avaliação destes objetos. Podemos encontrar na literatura diversas metodologias para analisar OA, sendo que nesta pesquisa os autores nấo apresentaram os critérios de escolha, mas utilizaram o método LORI para demonstrar como realizar a avaliação de dois OA.

Artigo 03: Uma abordagem para avaliação de jogos educativos: ênfase no ensino fundamental Ano de publicação: 2012

Autores: Maxwell de Oliveira Medeiros, Juliano Schimiguel

\section{Instituiçáo: Universidade Cruzeiro do Sul}

Síntese: Este artigo apresenta uma revisão bibliográfica sobre os benefícios e observaçóes importantes na utilização de jogos eletrônicos como ferramenta de apoio ao ensino e aprendizagem, principalmente no ensino fundamental. Os autores também realizam a avaliação de um jogo chamado "Stop!", para deixar como exemplo. Para isso, decidiram utilizar a combinação do método LORI com o GameFlow, pois o primeiro trata-se de uma ótima ferramenta para medir a qualidade de OA nos aspectos pedagógicos e técnicos, e o segundo trabalha apenas aspectos específicos para jogos, tais como: imersão e desafio. Nesta demonstraçáo, os autores têm o objetivo de auxiliar os professores a escolherem os tipos de jogos que mais atendam aos seus planos de ensino. 
Artigo 04: Análise da qualidade em objetos de aprendizagem: reflexão sobre aspectos pedagógicos Ano de publicaçáo: 2013

Autores: Laura A. F. Lima; Kátia M. Alonso; Cristiano Maciel

Instituiçáo: Universidade Federal do Mato Grosso

Síntese: Neste artigo, os autores trazem um estudo sobre padróes para avaliar adequadamente um $\mathrm{OA}$, observando critérios e aspectos pedagógicos presentes em instrumentos de avaliação. Os autores justificam: "Considerando que a real contribuição dos Objetos de Aprendizagem, no processo de ensino-aprendizagem do estudante, tem provocado inquietação em termos de qualidade em relaçáo a aspectos pedagógicos presentes em seu uso" (LIMA; ALONSO; MACIEL, 2013, p. 61). Segundo os pesquisadores, alguns destes aspectos foram definidos como: clareza de conteúdo, carga informacional adequada, identificação de pré-requisitos, atividades de reflexão, feedback claro e conciso, entre outros. Para a realização deste estudo, os autores utilizaram quatro metodologias de avaliação: o HEODAR (Herramienta de Evaluación de Objetos Didácticos de Aprendizaje Reutilizables), o CCEAD PUC-Rio, o NUTED (Núcleo de Tecnologia Digital Aplicada à Educação) e, obviamente, o LORI.

Artigo 05: Análise de métodos de avaliação de jogos educacionais

Ano de publicaçáo: 2014

Autores: Marcos Tsuda, Vinícius Moro Sanches, Thalles Gonçalves Ferreira, Joice Lee Otsuka, Delano Medeiros Beder

Instituiçáo: Universidade Federal de São Carlos

Síntese: Este trabalho apresenta um estudo sobre o funcionamento de quatro ferramentas para avaliação de objetos de aprendizagem: LORI, GameFlow, EGameFlow e UsaECG. Tendo como base procedimentos de pesquisa exploratória e bibliográfica, os autores realizaram grupos de pesquisa para avaliar quais destes métodos de avaliação seria o ideal para utilizar em dois jogos educacionais em desenvolvimento no Laboratório de Objetos de Aprendizagem da UFSCAR. Após a análise, os pesquisadores chegaram à conclusão que o método LORI e o GameFlow são métodos que possuem características que envolvem particularidades de jogos como, por exemplo, jogabilidade e imersão. Os autores concluem o artigo com as seguintes palavras: "Acreditamos que o uso desses instrumentos ao longo do processo de design, para validar desde as primeiras versôes funcionais dos jogos é bastante importante, possibilitando a identificação de problemas e refinamentos do jogo desde as fases iniciais de desenvolvimento (TSUDA et al. 2014, p. 166).

Artigo 06: Avaliação de jogos educativos: uma abordagem no ensino da matemática

Ano de publicaçáa: 2015

Autores: Wilk Oliveira dos Santos; Sebastião Rogerio da Silva Neto; Clovis Gomes da Silva Junior; Ig Ilbert Bittencourt

Instituiçáo: Universidade de Pernambuco; Universidade de Alagoas

Síntese: Os autores afirmam o seguinte: "A avaliação de jogos educativos é uma fase importante dentro de seu processo de desenvolvimento, por possibilitar averiguar o desempenho do jogo diante do seu 
público alvo" (SANTOS et al. 2015, p. 657). Diante disso, este trabalho tem por objetivo realizar a avaliação de dois jogos educativos, observando aspectos de interface e pedagógicos, combinando três metodologias de avaliação: LORI, GameFlow e Kirkpatrick. Os pesquisadores justificaram a escolha destes métodos da seguinte forma: o primeiro pode ser usado em qualquer $\mathrm{OA}$, mas, náo contempla itens específicos para jogos, o segundo avalia quão prazeroso é o jogo e o terceiro mede a eficiência da aplicação do jogo. Em suas considerações finais, os autores afirmam que ambos os jogos tiveram um bom resultado na maioria dos princípios observados, sendo que o método de avaliação triplo pode ser adaptado ou replicado para outros jogos educacionais.

Artigo 07: Validação de uma abordagem combinada para avaliação de software educativo: avanços e desafios

Ano de publicaçáo: 2016

Autores: Wendell S. Pereira; Raimundo J. Cardoso Filho; Williane Rodrigues de A. Silva; Raphael Salviano T. da Silva; Vanessa F. Dantas e Yuska P. C. Aguiar.

Instituição: Universidade Federal da Paraíba e Universidade de São Paulo

Síntese: Os autores afirmam que, diante de uma grande diversidade de metodologias para avaliação de OA, surgem dificuldades a respeito de qual técnica seria mais adequada. Diante disso, foi proposto um trabalho que promove a avaliaçáo de um objeto utilizando uma combinaçâo de vários métodos, com o propósito de identificar equivalências de critérios e possíveis falhas. Os métodos avaliados foram: Método de Reeves; TICESE; Técnica de Mucchielli; LORI; Metodologia de Martins; Método Rocha e Modelo de Avaliação de Campos. Nesta pesquisa, os autores afirmam que, para que uma análise seja completa, o instrumento de avaliação dever conter itens que verificam elementos pedagógicos, critérios de usabilidade e qualidade de software. Segundo os autores, em suas consideraçôes finais, a utilização de uma única metodologia de avaliação não contempla sozinha todos os critérios de qualidade exigíveis, portanto, é imprescindível a combinação de várias técnicas para aumentar a qualidade da análise.

Nesta análise qualitativa é possível observar a preocupação dos autores com a qualidade dos OA e como devem ser avaliados. Todos os trabalhos apresentam o método LORI como uma excelente ferramenta de apoio para avaliação dos objetos, entretanto, nem todos realizaram uma experiência, focando na revisão. Dentre os autores que demonstraram um exemplo prático, a maioria tratava-se de "jogos educacionais" e concluíram que seria importante combinar o método LORI com outro específico para contemplar aspectos específicos de jogos.

\section{Considerações finais}

Após realização da pesquisa dentro do universo do Google Acadêmico, pode-se encontrar sete artigos que foram julgados pertinentes ao nosso tema, ou seja, que utilizam o método LORI de alguma maneira como ferramenta de apoio a avaliaçáo de Objetos de Aprendizagem. Podemos observar no inventário que, apesar da importância de se analisar a qualidade de um OA, a quantidade de artigos 
encontrados nos últimos cinco anos que utilizam a ferramenta é muito inferior ao esperado, tendo em vista a credibilidade que diversos autores atribuem ao método. Ainda, durante a análise, vimos trabalhos realizados por diversos tipos de instituiçóes (Federal, Estadual, Particular e Confessional) e na maioria das regiốes do Brasil, porém, nem todos realizaram experiências empíricas com o método. Outro indicador que chama a atenção, foi o baixo número de artigos produzidos por pesquisadores da área da Educação, o que aponta para um baixo interesse de educadores nas questôes de avaliação de OA.

O LORI possui critérios interessantes para avaliar aspectos técnicos e pedagógicos de OA, contudo, na maioria dos artigos revisitados, os autores recomendam a combinação com outras técnicas de avaliação, principalmente em jogos educacionais, por envolver características peculiares. Até o momento, não foi encontrada nenhuma atualização para o método, e também não há dados sobre o assunto.

Ao final da pesquisa pode-se observar que, nos últimos anos, há uma crescente utilização de recursos computacionais para auxílio no ensino, que váo desde a uma simples apresentaçáo de vídeos e slides a um sofisticado jogo interativo. Contudo, sempre haverá a necessidade de avaliar se este recurso realmente atende aos requisitos básicos para contribuir com o aprendizado. Para isso, existem ferramentas mais simples para a realizar sua avaliação, como o LORI. Espera-se, portanto, que este artigo possa, de alguma maneira, auxiliar educadores, profissionais da informática e demais pessoas no estudo e reflexão sobre o tema. Nosso trabalho terá alcançado seus objetivos se conseguir disseminar a importância de se avaliar um OA antes de utilizá-lo para fins didáticos. Quiçá usando o LORI.

\section{Referências}

AGUIAR, E. V. B.; FLÔRES, M. L. P. Objetos de Aprendizagem: Teoria e Prática. In: TAROUCO, Liane Margarida Rockenbach et al. (org.). Objetos de Aprendizagem: Conceitos Básicos. Porto Alegre: Evangraf, 2014. p. 12-28.

ALMEIDA, R. R.; CHAVES, A. C. L.; ARAUJO JR., C. F. de. Avaliação de Objetos de Aprendizagem: Aspectos a Serem Considerados Neste Processo. III Simpósio Nacional de Ensino de Ciência e Tecnologia. Ponta Grossa: Anais... 2012.

BARBOSA, Gisele. Objetos de Aprendizagem como Recurso Educacional Digital para Avaliaçáo Financeira: Análise e Avaliação. 2014. 127 f. Dissertação de Mestrado - Universidade Federal de Juiz de Fora, Juiz de Fora, 2014.

BRAGA, Juliana C.; DOTTA, Silvia; PIMENTEL Edson; STRANSKY, Beatriz. Desafios para o Desenvolvimento de Objetos de Aprendizagem Reutilizáveis e de Qualidade. I WorkShop de Desafios da Computação Aplicada à Educação. Curitiba: Anais..., 2012.

BRAGA, Juliana C.; MENEZES, Lilian. Objetos de Aprendizagem: Introdução e Fundamentos. Santo André: UFABC, 2014.

BULEGON, A. M.; MUSSOI, E. M. Objetos de Aprendizagem: Teoria e Prática - Pressupostos Pedagógicos de Objetos de Aprendizagem. 1ª Edição. Páginas 75-54. Porto Alegre: Evangraf, 2014. 
CECHINEL, Cristian.; SÁNCHEZ-ALONSO, Salvador; SICILIA, Miguel-Ángel. Avaliação Automática da Qualidade de Objetos de Aprendizagem dentro de Repositórios. Revista Brasileira de Informática na Educaçáa, v. 20, n. 3, p. 44-59, 2012.

FARIA, Elaine T. O Professor e as Novas Tecnologias. In: ENRICONE, Délcia (Org.). Ser Professor. Porto Alegre, EDIPUCRS, 2004.p. 57-72.

GAMA, Carmen L. G. da. Método de Construçáo de Objetos de Aprendizagem com Aplicaçáo em métodos numéricos. 2007. 184 f. Tese de Doutorado - Universidade Federal do Paraná, Curitiba, 2007.

GUILlERMO, Oscar Eduardo P.; TAROUCO, Liane Margarida R.; Endres, Luiz Augusto M. Desenvolvimento de Objetos Educacionais: Experimentos em Hidráulica. Revista Renote Novas Tecnologias na Educaçáo, Porto Alegre, v. 3, n. 2, p. 388-399, 2005.

GALAFASSI, Fabiane P.; GLUZ, João Carlos; GALAFASSI, Cristiano. Análise Crítica das Pesquisas Recentes sobre as Tecnologias de Objetos de Aprendizagem e Ambientes Virtuais de Aprendizagem. Revista Brasileira de Informática na Educaçáo, v. 21, n. 3, p. 43-52, 2013.

LIMA, L. A. F.; ALONSO, K. M.; MACIEL, C. Análise da qualidade em Objetos de Aprendizagem: reflexão sobre aspectos pedagógicos. II Congresso Brasileiro de Informática na Educação. Campinas: Anais..., 2013. p. 61-70.

MEDEIROS, M. de O.; SCHIMIGUEL, J. Uma Abordagem Para Avaliação de Jogos Educativos: Ênfase no Ensino Fundamental. In: SIMPÓSIO BRASILEIRO DE INFORMÁTICA NA EDUCAÇÃO, 23., 2012. Rio de Janeiro: Anais... 2012.

PEREIRA, W. S.; CARDOSO FILHO, R. J.; SILVA, W. R. de A.; SILVA, R. S; T. da; DANTAS, V. F.; AGUIAR, Y. P. C. Validação de uma abordagem combinada para avaliação de software educativo: avanços e desafios. Revista Tecnologias na Educação (Ctrl+E), n. 8, v. 16, art. 7, p. 2016.

REATEGUI, E.; FINCO, M. D. Proposta de diretrizes para avaliação de Objetos de Aprendizagem considerando aspectos pedagógicos e técnicos. Revista Renote Novas Tecnologias na Educaçáo, Porto Alegre, v. 8, n. 3, p. 289-298, 2010.

SANTOS, M. E. K. L. dos; AMARAL, L. H. Avaliação de Objetos Virtuais de Objetos de Aprendizagem no Ensino da Matemática. REnCiMa, v. 3, n. 2, p. 83-93, 2012.

SANTOS, W. O.; SILVA NETO, S. R. da; SILVA JR, C. G. da; Bittencourt, I. I. Avaliação de jogos educativos: uma abordagem no ensino de matemática. XXVI Simpósio Brasileiro de Informática na Educação. Maceió: Anais... 2015. p. 657-666.

SILVA, R. S. T. da; SILVA, W. R. de A.; FILHO, R. J. C.; PEREIRA, W. S.; AGUIAR, Y. P. C.; DANTAS, V. F. Aplicação comparativa de diferentes abordagens de avaliação para o software educativo Duolingo: a complexibilidade de escolher uma abordagem adequada. Revista Tecnologias na Educaçáo (Ctrl+E), João Pessoa, n. 8, v. 16, art. 8, 2016.

TSUDA, M.; SANCHES, V. M.; FERREIRA, T. G.; OTSUKA, J. L.; BEDER, D. M. Análise de métodos de avaliaçấo de jogos educacionais. In: SBGAMES, 13. 2014. Porto Alegre: Anais..., 2014. p. 158-166. 


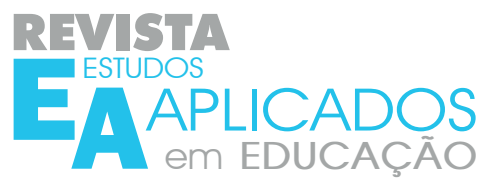

TAROUCO, L. M. R.; COSTA, V. M.; AVILA, B. G. Objetos de Aprendizagem: teoria e prática - projeto instrucional de Objetos de Aprendizagem. Porto Alegre: Evangraf, 2014. 